\title{
Cómo iniciar y mantener a más tenistas adultos aficionados en el tenis... El mejor deporte para la vida, la salud general y la aptitud física
}

\author{
Dave Miley
}

\section{RESUMEN}

Este artículo describe formas de iniciar el tenis de manera efectiva y mantener a los jugadores iniciantes / aficionados en el tenis. Describe los beneficios del tenis para la salud, probados científicamente, y muestra cómo utilizando las pelotas más lentas, los tenistas adultos iniciantes podrán pelotear más fácil y exitosamente, realizar una rutina de trabajo físico beneficiosa, comparable con el entrenamiento por intervalos, según las investigaciones presentadas. Afirma que gracias a las pelotas más lentas, ahora a los entrenadores les resulta más fácil adoptar la metodología del enfoque basado en el juego para iniciar a los adultos dentro de un entorno activo y dinámico de enseñanza. Este artículo diferencia entre "competición de tenis" y "juego de tenis", y enfatiza lo importante que es para los clubes y sedes de tenis utilizar ratings, formatos y puntajes adaptados para organizar de manera efectiva un "juego de tenis" social para los jugadores aficionados, y sugiere que esto se podría posicionar como un nuevo programa mundial de seguimiento para Tennis Xpress. Concentrándose en estos conceptos, los entrenadores y los centros de tenis podrán mantener a más tenistas adultos y proporcionarles un comienzo exitoso, positivo y divertido y con motivación para que continuen jugando y tratando de mejorar su nivel.
Palabras clave: adultos, tenis participación, recreativo, retención

Recibido: 30 Ene 2016

Aceptado: 25 May 2017

Autor correspondiente: Dave Miley.

Correo electrónico: dave.miley9@gmail.com

\section{INTRODUCCIÓN}

El tenis es uno de los mejores deportes. Sin embargo, las investigaciones actuales muestran que la tasa de participación en muchos de los países más desarrollados en el tenis está declinando significativamente (USTA, FFT, KNLTB, ITF). Este artículo hace referencia a algunos modos efectivos para atraer y mantener a los adultos en el tenis.

\section{BENEFICIOS DEL TENIS: QUÉ DICE LA CIENCIA}

Hay muchas investigaciones publicadas que demuestran los beneficios de jugar al tenis.
Dr. Jack Groppel, Co-fundador del Instituto de Rendimiento Humano Johnson y Johnson, en un artículo publicado en 1998, mencionaba las "34 Razones para jugar al tenis" y su efecto favorable, tanto para la salud como para la aptitud física, aún para los jugadores aficionados.

Otros estudios han ampliado lo afirmado por el Dr. Groppel en sus artículos. Pluim y cols. (2007) demostraron que los jugadores aficionados que juegan una hora de tenis con jugadores de nivel similar, cubren una distancia media de 3.17 kilómetros y logran una frecuencia cardíaca media de 149 latidos por minuto, mientras juegan.

Una investigación más a fondo llegó a la conclusión de que existía una asociación positiva entre una participación en el 
tenis regular y un mejor estado aeróbico, un cuerpo más magro, un perfil lípido favorable, una mejor salud ósea, y un menor riesgo de morbidez y mortalidad cardiovascular.

Kovacs y cols. (2016) concluyen que la participación en el tenis regular puede mejorar la capacidad aeróbica, disminuir la frecuencia cardíaca y la presión sanguínea en reposo, mejorar la densidad ósea, el tiempo de reacción, la fuerza y la flexibilidad, y disminuir la grasa corporal, y el riesgo cardiovascular y de mortalidad.

Otra investigación evaluó a personas que practicaron diferentes deportes durante 20 años, y concluyó que jugando tenis o bádminton, tres veces por semana, se puede reducir el riesgo de enfermedad coronario un $56 \%$ (Oja y cols., 2016).

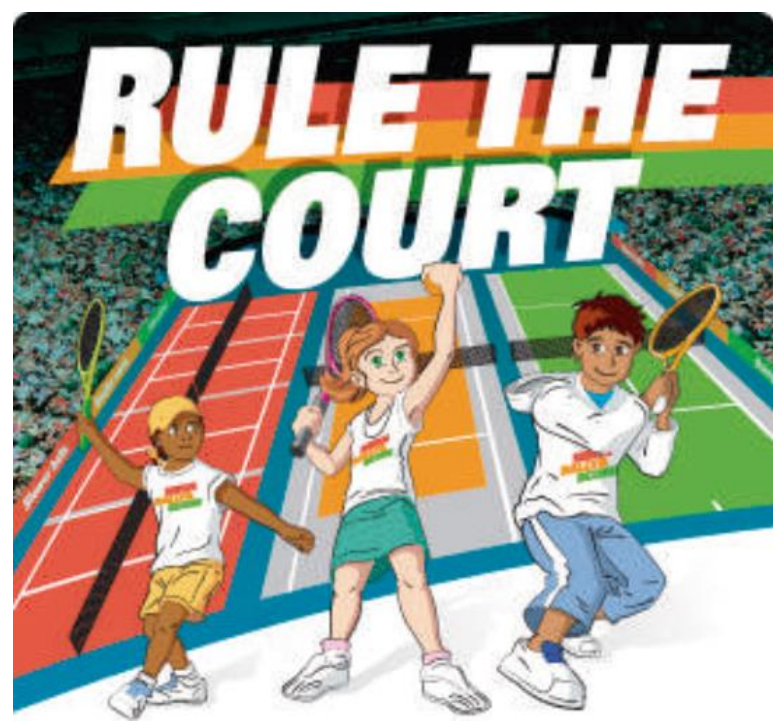

¡No hay ninguna duda de que jugar al tenis a nivel recreativo es excelente para la salud y la aptitud física!

\section{¡LA PROMOCIÓN EFECTIVA DEL TENIS NO ES SUFICIENTE!}

La promoción de los probados beneficios del tenis es muy importante, como lo son el servicio al cliente, la animación y la pasión que transmitan los entrenadores. Sin embargo, la promoción y el mejor servicio al cliente en el deporte no proporcionarán la solución para los retos de la participación si el producto que obtienen los clientes en su primera exposición al tenis no les motiva para regresar.

Hay muchos ejemplos de programas adultos bien promocionados por la Asociación Nacional que han atraído exitosamente a los adultos, pero con escasas tasas de retención asociada, como en el caso del programa bien promocionado de Centros de Bienvenida al Tenis de la USTA en 2008. Si el deporte es tan bueno, ¿por qué son tan bajas las tasas de retención de adultos?

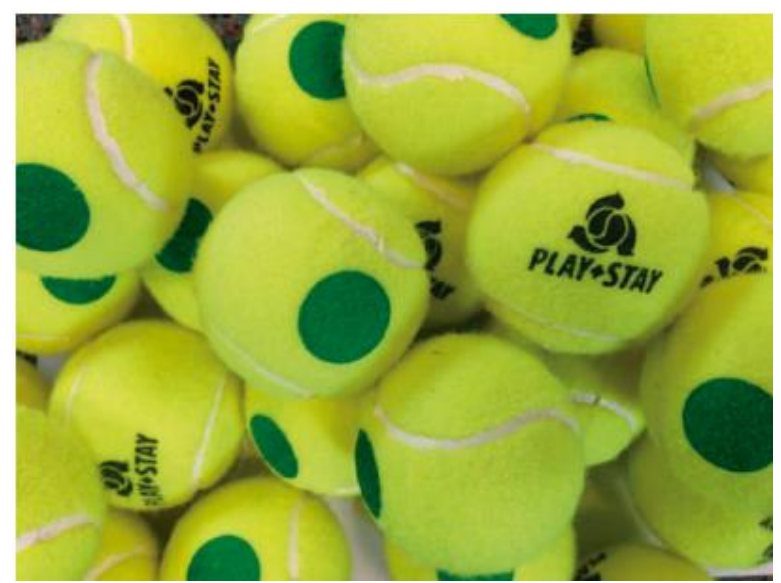

¡PELOTAS MÁS LENTAS, CANCHAS MÁS PEQUEÑAS, JUEGO FÁCIL!

Comencemos viendo cómo ha cambiado la enseñanza a los niños. Participé de la tan exitosa campaña Tennis Play and Stay de la ITF, lanzada en 2007. Esta campaña iniciada, con la ayuda de expertos en participación en el tenis, con la colaboración de la industria del tenis, y con el apoyo de los Circuitos de la ATP y de la WTA, llevó al subsiguiente cambio de reglas que manda el uso de las pelotas más lentas para la competición de los menores de 10 años. Esto cambió radicalmente, y en todo el mundo, la forma de iniciación del tenis a los niños menores. Si las competiciones usaban estas pelotas, los entrenadores debían utilizarlas para el entrenamiento. Lo obvio suele ser el secreto más grande, y hoy, la mayoría de los entrenadores de todo el mundo está utilizando las pelotas más lentas con raquetas y canchas modificadas, y haciendo el juego más fácil y más divertido para los niños (Reid y Farrow, 2010).

Las pelotas más lentas también permitieron un cambio en la metodología de enseñanza, simplificando a los entrenadores el cambio desde una metodología de iniciación, generalmente cargada de instrucción técnica, situaciones de enseñanza relativamente estáticas y con poco juego, hacia un enfoque basado en el juego, en el cual los niños juegan el juego, o deben realizar tareas activas basadas en éste. Los entrenadores, entonces, proporcionan la instrucción pertinente y utilizan las pelotas más lentas para diferenciar las tareas y asegurar el éxito.

\section{EL TENIS NECESITA COMPARARSE CON OTROS DEPORTES}

Si bien la iniciación del tenis a los niños ha cambiado radicalmente, la mayoría de los entrenadores del mundo continúa enseñando el tenis a los adultos, utilizando la pelota estándar, en un entorno de enseñanza relativamente estático y generalmente demasiado técnico.

Al llegar a los 35-40 años de edad, la mayoría abandona o reduce significativamente su participación en otros deportes, y muchos se vuelcan en el tenis. Sin embargo, la primera experiencia para los adultos suele dedicar algún tiempo, en el 
mejor de los casos, al peloteo, al movimiento, a jugar el juego y a hacer una rutina de ejercicio. Estos adultos suelen venir de la práctica de deportes activos y dinámicos con una buena rutina de ejercicios, razonable éxito y diversión. ¡El tenis necesita compararse bien con el deporte activo de donde vienen!

La mejor parte, y la más adictiva del tenis es golpear, devolver la pelota sobre la red, y luego jugar el punto...jSacar, Pelotear y Jugar puntos! Esto es lo que logró enganchar a la mayoría de los tenistas, y si se logra dar a los jugadores iniciantes ese "gusto" por lo mejor del tenis lo antes posible, hay más probabilidades de mantenerles.

\section{LAS PELOTAS LENTAS.... ¡NO SON SOLAMENTE PARA LOS NIÑOS!}

Como parte de la campaña Play and Stay, se lanzó Tennis Xpress en 2012, alentando a los entrenadores a usar las pelotas más lentas también con los adultos iniciantes. El programa inicial que usa las pelotas más lentas en un entorno de enseñanza activo y dinámico para presentar el juego, ha demostrado ser muy efectivo para enseñar el tenis a los adultos (ej. El exitoso programa Tennis Xpress de la LTA, lanzado en 2013).

Los adultos de hoy tienen cortos espacios de atención cuando aprenden algo nuevo, y están acostumbrados a lograr "éxito" rápidamente en sus actividades durante el tiempo libre. Es clave que la experiencia para los tenistas iniciantes sea, no solamente activa y divertida, sino que implique cierto éxito razonable desde esa primera lección.

Jamás encontré un adulto iniciante que no pueda pelotear, con por lo menos una de las pelotas más lentas rojas, naranja o verdes. El uso de las pelotas más lentas asegura que los iniciantes pueden pelotear con otros iniciantes, y garantiza más éxito, más peloteos, más diversión y un gran trabajo físico. En cuanto a la investigación citada, los jugadores iniciantes peloteando con las pelotas más lentas, se mueven 1 o 2 metros en diferentes direcciones, para llegar a la pelota con una frecuencia cardíaca que supera los 140 latidos por minuto. Hacen entrenamiento por intervalos $y$, al mismo tiempo, se divierten.

Tennis Xpress ha tenido éxito, pero aún debe lograr ese grado de impacto global que el mandato de la competición de menores de 10 años tuvo en la forma de trabajar de los entrenadores con los niños menores. Como no existe una reglamentación implementada para los entrenadores que trabajan con adultos iniciantes, se necesita una extensa promoción para cambiar la conducta del entrenador.

\section{JUEGO ADECUADO /LA COMPETICIÓN IMPULSA EL DEPORTE}

Una vez que los jugadores se inician en el tenis, y deciden continuar en el deporte, el tenis se enfrenta a otro desafío para motivar y para que permanezcan en el juego. Suele decirse que "la competición impulsa el deporte". Creo que es más correcto decir que "el juego apropiado/la competición apropiada impulsa el deporte".

Para mí, existe una diferencia entre "juego" y "competición". La palabra "juego" se asocia con diversión. La "competición" es más seria y puede intimidar a los jugadores aficionados. Los resultados de la "competición en el tenis" generalmente se registran. En el "juego de tenis" llevas el puntaje y juegas el juego, pero los resultados no se registran, por lo cual, hay menos presión y más probabilidades de ganar.

Muchos centros de tenis, hoy, según mi opinión, están impulsados por demasiado entrenamiento. Suelen ser buenos para organizar el entrenamiento, pero en general proporcionan oportunidades competitivas solamente para un número pequeño de jugadores más avanzados. No son tan buenos para organizar oportunidades de "juego" para los jugadores aficionados. Para realmente mantener a los jugadores aficionados iniciantes, es importante no solamente la introducción efectiva, sino también adaptar el seguimiento del "juego de tenis" a las necesidades y estilos de vida de los jugadores aficionados, organizando oportunidades de juego amistosas en combinación con las pelotas más lentas. Idealmente, el "juego" debe involucrar un elemento social, en el cual los participantes se encuentren y mezclen con otros jugadores aficionados y que se lleve a cabo en un momento apropiado para los jugadores en cuestión (ITF Tennis Xpress, lo que desean los adultos, 2012). Quizás un nuevo programa "Tennis Xpress Play" ¿podría ser un seguimiento para Tennis Xpress?

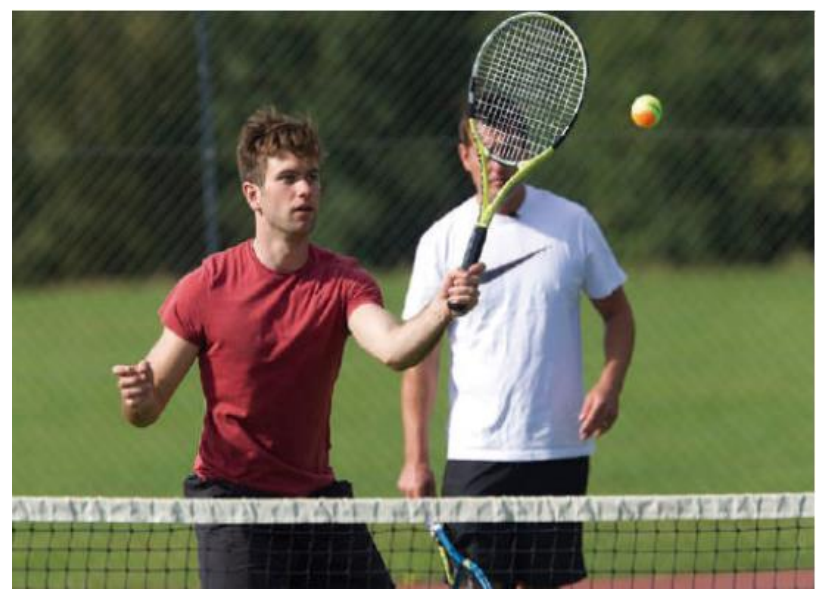

Las Reglas de Tenis permiten muchos formatos y sistemas de puntaje diferentes que se pueden utilizar de manera efectiva para una mejor retención de los jugadores aficionados (Crespo y Miley, 1999). Permiten el uso de la pelota verde, que se asemeja a la pelota amarilla estándar y en la cancha total. La disponibilidad de esta pelota para el juego recreativo de los adultos es otro desafío. Los fabricantes y las federaciones nacionales de tenis necesitan trabajar juntos para no solo promover de manera efectiva la pelota verde, sino también para mejorar su disponibilidad en negocios minoristas y centros de tenis. 


\section{CLASIFICACIONES (RATINGS)}

La introducción y el uso de clasificaciones para todos los jugadores aficionados también puede ser una herramienta muy efectiva en el nivel micro / club. Una vez que un jugador ha iniciado y ha progresado, y juega con la pelota verde o la pelota amarilla estándar, deberá recibir una clasificación del club para poder encontrar jugadores de similar nivel con quienes jugar, $y$ de este modo, transitar un camino para continuar jugando y mejorar.

\section{TENNIS CANADA - ¡UN GRAN EJEMPLO DE BUENA PRÁCTICA!}

Uno de los pocos países, entre los más desarrollados en tenis, que ha notado incrementos significativos en participación, es Canadá (el número de tenistas jugando, por lo menos una vez, pasó de 5.055 .000 en 2012, a 6.532 .000 en 2016 (Charlton Strategic Research Inc., 2016).

\section{CONCLUSIÓN}

El tenis ha demostrado ser uno de los mejores, o, el mejor deporte para toda la vida, y para la salud y aptitud física. Hoy en dia, hay herramientas para que la iniciación al tenis de los jugadores adultos iniciantes sea más activa y dinámica, proporcionando un éxito razonable, y una buena rutina de ejercicio jugando el juego desde la primera lección.

Los entrenadores deben usar las pelotas más lentas y la metodología basada en el juego para asegurar una iniciación al juego activa, exitosa y divertida. El centro de tenis deberá proporcionar, para los jugadores aficionados adultos, formatos de juego y de puntaje adaptados, en un entorno social amistoso, utilizando la pelota verde, junto con clasificaciones para mantener mejor a los jugadores que se iniciaron.

Si los centros de tenis y los entrenadores de todo el mundo se centran en estas herramientas importantes para atraer $y$ mantener a los jugadores iniciantes/ aficionados, y el tenis continúa promocionando sus beneficios positivos de manera efectiva, ¡creo que no habrá límites para el desarrollo del deporte a nivel mundial!

\section{REFERENCIAS}

Charlton Strategic Research Inc. (2016). Tennis Canada Participation statistics.

Crespo, M., and Miley, D. (1999). ITF Competition Formats Manual. London. ITF Ltd

Farrow, D., \& Reid, M. (2010). The effect of equipment scaling on the skill acquisition of beginning tennis players. Journal of Sports Sciences, 28(7) 723-732. https://doi.org/10.1080/02640411003770238
Fernandez-Fernandez, J., Sanz-Rivas, D., Sanchez-Muñoz, C., Pluim, B. M., Tiemessen, I., \& Mendez-Villanueva, A. (2009). A comparison of the activity profile and physiological demands between advanced and recreational veteran tennis players. The Journal of Strength \& Conditioning Research, 23(2), 604-610. https://doi.org/10.1519/JSC.0b013e318194208a

Groppel, J. (1998). Tennis for the Health of it. 34 Reasons to Play Tennis. USPTA.

ITF. (2000). Research Participation Surveys, 2000-2012.

ITF. (2010). Tennis 10s promotional leaflets and implementation manual.

ITF. (2010). Tennis Xpress promotional leaflets and implementation manual.

Kovacs M, Pluim B, Groppel J, Crespo M, Roetert P, Hainline B, Miller S, Reid M, Pestre B, De Vylder M, Dunn N, Miley D, Jones T. (2016). Health Wellness and Cognitive Performance Benefits of Tennis. Journal of Medicine and Science in Tennis.

Oja, P., Kelly, P., Pedisic, Z., Titze, S., Bauman, A., Foster, C., ... \& Stamatakis, E. (2016). Associations of specific types of sports and exercise with all-cause and cardiovascular-disease mortality: a cohort study of 80306 British adults. Br J Sports Med, bjsports2016. https://doi.org/10.1136/bjsports-2016-096822

Pluim, B. M., Staal, J. B., Marks, B. L., Miller, S., \& Miley, D. (2007). Health benefits of tennis. British journal of sports medicine.431. https://doi.org/10.1136/bjsm.2006.034967

Statistical research on participation in USA, Netherlands, and France, USTA, TIA Talking Points report, Sports Marketing Surveys, 2016; KNLTB, Membership figures and Mulier Institute report 2016; FFT membership figures and club participation report, 2016.

\section{CONTENIDO ITF ACADEMY RECOMENDADO (HAZ CLICK ABAJO)}

\section{ITF ${ }^{\circ}$ Academy}

Derechos de Autor (c) 2017 Dave Miley

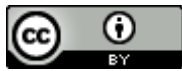

Este texto está protegido por una licencia CreativeCommons 4.0 .

Usted es libre para Compartir -copiar y redistribuir el material en cualquier medio o formato- y Adaptar el documento - remezclar, transformar y crear a partir del material- para cualquier propósito, , incluso para fines comerciales, siempre que cumpla la condición de:

Atribución: Usted debe dar crédito a la obra original de manera adecuada, proporcionar un enlace a la licencia, e indicar si se han realizado cambios. Puede hacerlo en cualquier forma razonable, pero no de forma tal que sugiera que tiene el apoyo del licenciante o lo recibe por el uso que hace de la obra. 